\title{
Respiratory Distress, Congenital Hypothyroidism and Hypotonia in a Newborn
}

\author{
Sophie Jovien ${ }^{a}$ Raphaël Borie ${ }^{b, c, d}$ Diane Doummar ${ }^{e}$ Annick Clement ${ }^{a, f}$ \\ Nadia Nathan ${ }^{\mathrm{a}, \mathrm{f}}$ \\ a Service de Pneumologie Pédiatrique, Hôpital Armand Trousseau, Assistance Publique Hôpitaux de Paris, \\ Centre national de référence des maladies respiratoires rares RespiRare, ${ }^{b}$ Service de Pneumologie A, Hôpital Bichat, \\ Assistance Publique Hôpitaux de Paris, Centre de compétence des maladies pulmonaires rares, 'Université Paris \\ Diderot, Sorbonne Paris Cité, dDHU FIRE, e Service de Neurologie Pédiatrique, Hôpital Armand Trousseau, Assistance \\ Publique Hôpitaux de Paris, and f Inserm UMRS933, Université Pierre et Marie Curie (Paris 6), Sorbonne Universités, \\ Paris, France
}

A male infant was born at term and eutrophic at birth. On day 6 , he developed severe respiratory distress that required non-invasive ventilation support and oxygen therapy. Clinical examination revealed axial hypotonia, congenital myxoedema, cutaneous jaundice, large fontanels, and mild macroglossia. The diagnosis of hypothyroidism was confirmed by the neonatal systematic screening results, with an absence of thyroid tissue. Chest high-resolution computed tomography (HRCT) showed diffuse alveolar and interstitial opacities (fig. 1a).

The child was supplemented with L-thyroxine. Noninvasive ventilation could be stopped after a few days, but he remained hypoxic with $73 \mathrm{~mm} \mathrm{Hg} \mathrm{PaO}_{2}$ under 1 litre/ min oxygen supplementation. At the age of 3 months, oral long-term azithromycin was started followed by intravenous monthly corticosteroid pulses $\left(300 \mathrm{mg} / \mathrm{m}^{2} /\right.$ day for 3 days) and oral corticosteroids [1]. At the age of 10 months, his clinical evaluation was stable. A HRCT scan showed a diffuse increase in the ground-glass attenuations. Oral hydroxychloroquine was added but had to be stopped after 4 months because of neutropenia. Progressively, under corticosteroid pulses and azithromycin, his respiratory status improved, and at the age of 2 years, oxygen therapy was discontinued. Neurological evaluation documented an important motor and speech delay with hypotonia and choreic movements.

The patient's mother, aged 26 years, reported a medical history of abnormal movements since the age of 5 years and chronic cough during childhood, with no proven aetiology. Her clinical evaluation documented benign hereditary chorea appearing in infancy, which had improved up to early adulthood and evolved to myoclonus. She presented compensated hypothyroidism.

Surprisingly, despite the absence of complaints, respiratory investigations revealed stage II NYHA dyspnoea, hypoxemia with $57 \mathrm{~mm} \mathrm{Hg} \mathrm{PaO}$, and a severe restrictive pattern with diffusion impairment on lung function tests. A HRCT scan showed diffuse ground-glass attenuations, sub-pleural cysts and mild lung fibrosis (fig. 1b). A left to right spontaneous massive shunt through the patent foramen ovale was discovered. After percutaneous closure, her respiratory condition improved partially. She was put under azithromycin treatment for 3 months, then continuous oral corticosteroid for only 1 month without any objective improvement. The treatment was recently replaced by hydroxychloroquine.

What is your diagnosis?

\section{KARGER}

E-Mail karger@karger.com www.karger.com/res
(C) 2016 S. Karger AG, Basel

0025-7931/16/0923-0188\$39.50/0
Dr. Nadia Nathan

Service de Pneumologie Pédiatrique, Hôpital Armand Trousseau 26 avenue du Dr Arnold Netter FR-75012 Paris (France)

E-Mail nadia.nathan@aphp.fr 

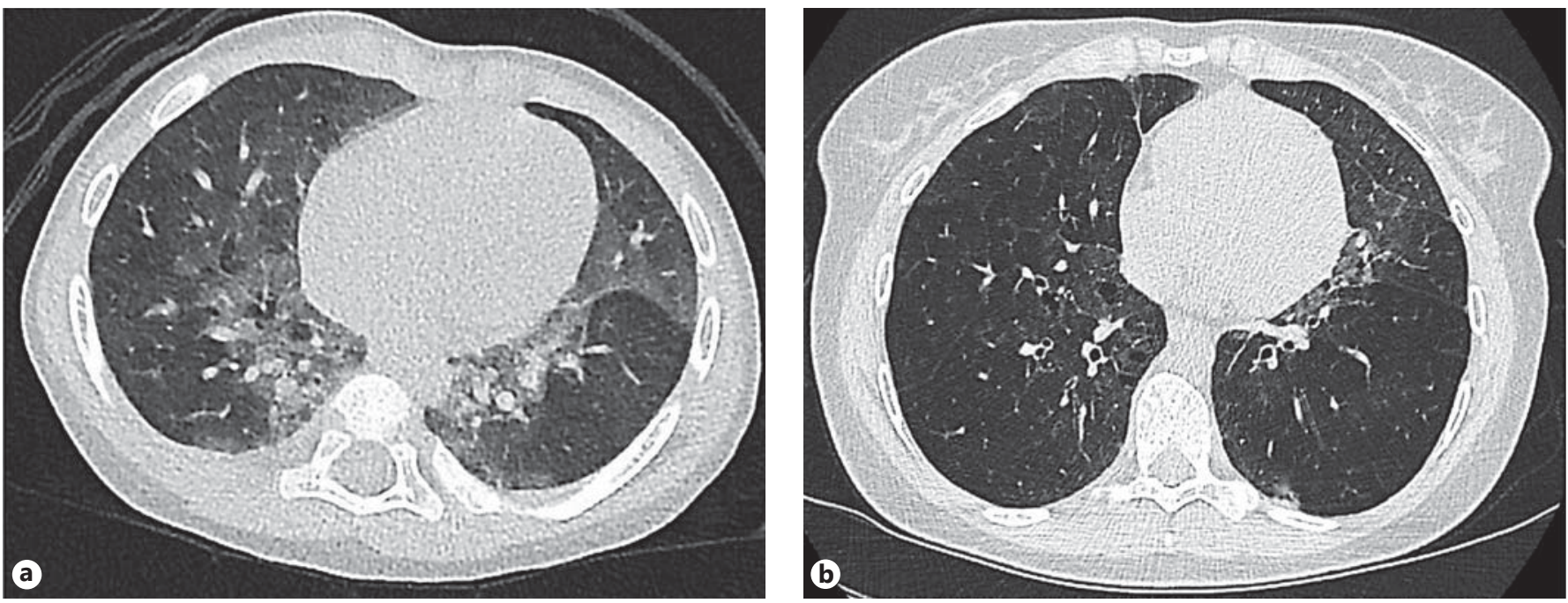

Fig. 1. HRCT scan of the two related patients. a Chest HRCT scan of the infant (individual III.2, fig. 2) at the age of 16 months showing diffuse ground-glass attenuations. b Chest HRCT scan of the mother (individual II.3, fig. 2) at the age of 27 years showing a fibrosing interstitial pattern with bilateral septal thickening and mild groundglass attenuations. 


\section{Diagnosis: Familial 'Brain-Lung-Thyroid Syndrome' Related to a NKX2-1 Mutation}

Surfactant protein disorders were suspected and analysis of the NK2 homeobox 1 gene (NKX2-1, OMIM 610978), also known as thyroid transcription factor 1 (TTF1), revealed the presence of a novel splicing mutation: c.373+2T>C, NM_003317.3. Following the identification of the NKX2-1 mutation in the baby, a familial screening was proposed, which led to the identification of the NKX21 c. $373+2 \mathrm{~T}>\mathrm{C}$ novel mutation in the mother (fig. 2).

NKX2-1 (NM_003317.3) heterozygous mutations have been associated with 'brain-lung-thyroid syndrome' with variable degrees of interstitial lung disease (ILD), thyroid dysfunction (hypothyroidism) and neurological abnormalities (hypotonia and benign chorea) [2-7].

This family illustrates well the large phenotype variability of the disease. Whereas the lung disease started early in life and was severe in the child, the mother presented moderate childhood respiratory disease and evolved towards lung fibrosis in adulthood. The benign chorea was predominant in her and appeared later than usually described, with compensated hypothyroidism $[6,8,9]$. Depending on the series, complete brain, lung and thyroid disease is described in $30-50 \%$ of patients with NKX2-1 mutation $[5,8,10]$. Isolated benign chorea or lung involvement have also been described in $13-24 \%$ of cases $[4,5,8,10]$. The reason why patients display such heterogeneous phenotypes for the same NKX2-1 mutation is unclear. Various types of molecular defects have been described without phenotype correlations, except for whole-gene deletion that may be associated, unexpectedly, with milder pulmonary disease [4].

In France, systematic neonatal screening for hypothyroidism can lead to the early diagnosis of 'brain-lung-thyroid syndrome'. However, considering the high frequency of incomplete forms of the disease, even in the absence of hypothyroidism, unexplained ILD in a young patient should justify a NKX2-1 analysis [5].

Corticosteroids are the mainstay of the ILD treatment in children with various surfactant disorders, including NKX2-1 mutations. Other medications have been used, such as azithromycin for its immunomodulatory properties or immunosuppressive drugs, including hydroxychloroquine $[1,11]$.

Familial cases of 'brain-lung-thyroid syndrome' are rare, with highly heterogeneous phenotypes, even in a single family. As clinical manifestations can appear over time, an isolated organ disease may not exclude the diagnosis. Therefore, a multidisciplinary management with lung physicians, endocrinologists and neurologists is recommended to propose an early treatment.

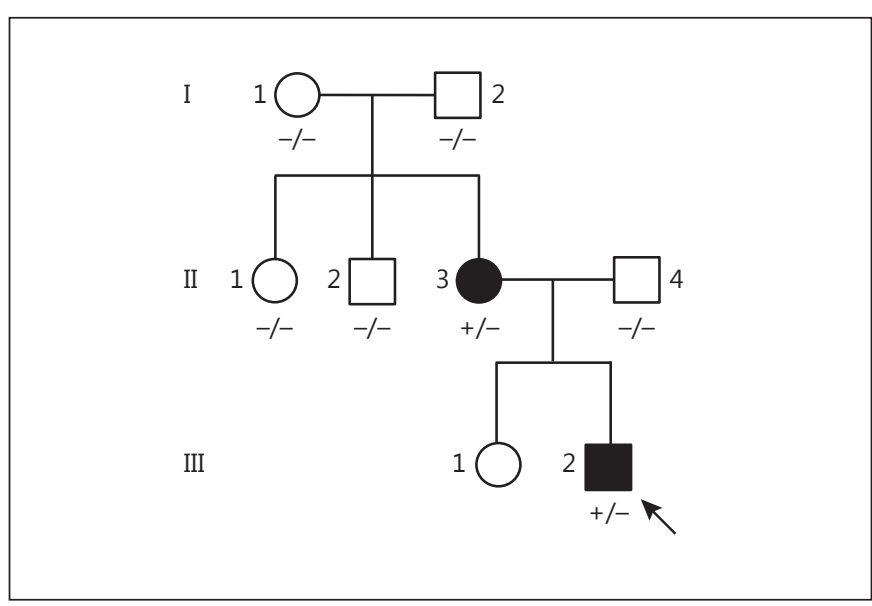

Fig. 2. Pedigree of the described family. The index case III.2 (arrow) was diagnosed with 'brain-lung-thyroid syndrome' (black symbol). A heterozygous (+/-) novel splicing NKX2-1 (NM_003317.3) mutation c.373+2T >C was identified for him and secondarily for his mother (II.3). Both of the mother's parents (I.1 and I.2) presented neither hypothyroidism nor neurological or respiratory symptoms. Chest CT scans were normal. None of them carried the NKX2-1 mutation (-/-). The neomutation was thus confirmed in the mother.

\section{Acknowledgements}

The authors thank the patients and their families for their agreement to publication and their reviewing of the manuscript. They also thank the patient association 'Ensemble pour Pedro' (http://ensemblepourpedro.simplesite.com/) as well as the clinicians and the biologist who are in charge of the patient and contributed to his diagnosis: Estelle Breton, MD, Mélanie Henriat, $\mathrm{MD}$, Julie Beucher, MD, PhD, and Malek Louha, MD.

\section{Statement of Ethics}

The authors confirm that they obtained written informed permission from the adult patient and from the child's guardian for publication in Respiration.

\section{Disclosure Statement}

The authors declare that they have no financial or other conflict of interest.

\section{Key Words}

NKX2-1 - TTF1 - Interstitial lung disease · Benign hereditary chorea $\cdot$ Hypothyroidism 


\section{References}

$>1$ Bush A, Cunningham S, de Blic J, Barbato A, Clement A, Epaud R, et al: European protocols for the diagnosis and initial treatment of interstitial lung disease in children. Thorax 2015;70:1078-1084.

$>2$ Willemsen MAAP, Breedveld GJ, Wouda S, Otten BJ, Yntema JL, Lammens $M$, et al: Brain-thyroid-lung syndrome: a patient with a severe multi-system disorder due to a de novo mutation in the thyroid transcription factor 1 gene. Eur J Pediatr 2005;164:28-30.

3 Iwatani N, Mabe H, Devriendt K, Kodama M, Miike T: Deletion of NKX2.1 gene encoding thyroid transcription factor-1 in two siblings with hypothyroidism and respiratory failure. J Pediatr 2000;137:272-276.

$\checkmark 4$ Hamvas A, Deterding RR, Wert SE, White FV, Dishop MK, Alfano DN, et al: Heterogeneous pulmonary phenotypes associated with mutations in the thyroid transcription factor gene NKX2-1. Chest 2013;144:794-804.
5 Carré A, Szinnai G, Castanet M, Sura-Trueba S, Tron E, Broutin-L'Hermite I, et al: Five new TTF1/NKX2.1 mutations in brain-lung-thyroid syndrome: rescue by PAX8 synergism in one case. Hum Mol Genet 2009;18:22662276.

6 Williamson S, Kirkpatrick M, Greene S, Goudie D: A novel mutation of NKX2-1 affecting 2 generations with hypothyroidism and choreoathetosis: part of the spectrum of brain-thyroid-lung syndrome. J Child Neurol 2014;29:666-669.

7 Guillot L, Carre A, Szinnai G, Castanet M, Tron E, Jaubert F, et al: NKX2-1 mutations leading to surfactant protein promoter dysregulation cause interstitial lung disease in 'brain-lung-thyroid syndrome'. Hum Mutat 2010;31:E1146-E1162.
8 Gras D, Jonard L, Roze E, Chantot-Bastaraud S, Koht J, Motte J, et al: Benign hereditary chorea: phenotype, prognosis, therapeutic outcome and long term follow-up in a large series with new mutations in the TITF1/ NKX2-1 gene. J Neurol Neurosurg Psychiatry 2012;83:956-962.

9 Peall KJ, Kurian MA: Benign hereditary chorea: an update. Tremor Other Hyperkinet Mov (N Y) 2015;5:314.

-10 Veneziano L, Parkinson MH, Mantuano E, Frontali M, Bhatia KP, Giunti P: A novel de novo mutation of the TITF1/NKX2-1 gene causing ataxia, benign hereditary chorea, hypothyroidism and a pituitary mass in a UK family and review of the literature. Cerebellum 2014;13:588-595.

11 Parnham MJ, Erakovic Haber V, Giamarellos-Bourboulis EJ, Perletti G, Verleden GM, Vos R: Azithromycin: mechanisms of action and their relevance for clinical applications. Pharmacol Ther 2014;143:225-245. 